\title{
Cooling and reduced risk of clotting within the extracorporeal continuous renal replacement circuit
}

Received: 25 September 2008

Accepted: 25 September 2008

Published online: 8 October 2008

(C) Springer-Verlag 2008

This editorial refers to the article available at: doi:10.1007/s00134-008-1271-9.

\section{S. M. Bagshaw (匹)}

Division of Critical Care Medicine, University of Alberta Hospital, University of Alberta, 3C1.16 Walter C. Mackenzie Centre,

8440-122 Street, Edmonton, AB T6G2B7, Canada

e-mail: bagshaw@ualberta.ca

Tel.: +1-780-4076755

Fax: +1-780-4071228

\section{A. Davenport}

UCL Center for Nephrology,

Royal Free and University College Medical School, London, UK

Clotting of the extracorporeal circuit, with a corresponding loss of blood in the circuit remains the Achilles heel of continuous renal replacement therapies (CRRT) [1]. Pre-mature circuit clotting results not only in a loss of efficacy of treatment, but also an increase in nursing work load, and higher financial costs due to circuit replacement $[2,3]$.

Early circuit clotting may be the consequence of numerous factors including bio-incompatibility, inadequate vascular access, and critical illness in general (including thrombin generation, thrombocytosis, blood transfusions etc.) [4-8], along with specific CRRT circuitrelated factors such as stasis of blood flow [7, 9], hemoconcentration [7], and blood-air contact in air-detection chambers [7].

Hypothermia has long been known to affect coagulation $[10,11]$ and therapeutic hypothermia may useful for reducing the risk of thrombus formation [12]. These observations are further supported by data showing a reduced pro-coagulant response to recombinant activated factor VII when administered in severe hypothermia [1315].

Based on these observations, Krouzecky and colleagues now report on the deliberate cooling of blood in the extracorporeal CRRT circuit down to a temperature of $20^{\circ} \mathrm{C}$ as a novel technique for regional anticoagulation in an experimental porcine model [16]. This study randomized 12 anesthetized and ventilated pigs to $6 \mathrm{~h}$ of CRRT (neutral fluid balance, $20 \mathrm{~mL} / \mathrm{kg}$ per h) with either regional blood cooling of the extracorporeal circuit (hypothermic circuit) or control (isothermic circuit). No anticoagulation was used. Regional temperature manipulation in the extracorporeal circuit was achieved by use of a cooling exchange device at the blood inflow tract (set at $20^{\circ} \mathrm{C}$ ) and a concomitant warming exchange device at the blood return tract (set at $38^{\circ} \mathrm{C}$ ), both of which were monitored and controlled by a central computer. Numerous parameters related to circuit patency (i.e. time to clotting, alarm-trigger pump stopping, transmembrane pressure) and coagulation status [i.e. thrombin-antithrombin complexes (TAT), thromboelastography (TEG)] were determined before, during and following the prescribed therapy. The authors also investigated whether regional blood cooling altered systemic hemodynamics or induced significant hemolysis compared with isothermic controls. The key finding of the study was that regional blood cooling was associated with patency of all the extracorporeal circuits for the duration of applied CRRT, whereas five of the six isothermic circuits prematurely clotted. The clotting in the isothermic circuits was associated with a significant increase in transmembrane pressures and an increase in detectable extracorporeal TAT complexes, compared to the hypothermic circuits. In addition, blood sampling from the hypothermic circuits showed evidence of a significant delay in clotting time and a decrease clotting rate when assessed by standardized TEG, a functional assay of clot formation and lysis. 
Finally, the study reported no important differences in systemic hemodynamics, platelet count or hemolysis between the treatment groups.

It is important to recognize that the extracorporeal circuit does not contain the endothelial anti-thrombus regulators: nitric oxide, prostacyclin and ectonucleases. While factor XII, the initiating factor of the extrinsic coagulation pathway, is deposited on the dialyzer/hemofilter membrane, activation of this coagulation cascade is not necessarily required for membrane clotting, as factor XII is deposited along with its natural inhibitor [17]. Endothelial damage typically leads to collagen exposure and release of tissue factor to initiate local clotting. Conversely, in the extracorporeal circuit, tissue factor is released following the activation of leukocytes and monocytes by the dialyzer/hemofilter membrane, leading to budding and shedding of cell surface membrane. Tissue factor release generates thrombin, which not only converts fibrinogen to fibrin, but also activates platelets, and contributes to platelet micro-emboli and further thrombin generation [1].

There would appear adequate biologic rationale and experimental data to support the potential of blood cooling to regionally anticoagulate the extracorporeal circuit during CRRT. Cooling affects the fluid dynamics of cell membrane lipid bilayers, reducing cell blebbing and shedding of lipid rich surface fragments, and so reducing tissue factor release and thrombin generation. Similarly, platelet function (i.e. aggregation and adhesion) is similarly known to be adversely affected by hypothermia [18] From these data, we are unable to comment specifically on the impact of cooling on platelet function, however, both groups showed significant reductions in platelet count during the study suggesting a consumptive process. The standardized TEG reaction time (measure of fibrin formation) and time for TEG tracing to reach amplitude of $20 \mathrm{~mm}$ (measure of dynamic clot formation) were most affected by the regional blood cooling, while there was no significant difference in the overall final width of the TEG tracing at $60 \mathrm{~min}$ (measure of clot stability). If the TEG had been adjusted to the temperature of the circuit, then there may have possibly been a reduction in the TEG maximal amplitude.

Interestingly, these effects of blood cooling on reducing clotting of the extracorporeal CRRT circuits may, in part, explain some of the circuit survival advantages reported for both pre-dilutional hemofiltration fluid replacement [19] and also during spontaneous arteriovenous hemofiltration when compared to pump-driven veno-venous circuits [20, 21]. Both pre-dilutional fluid replacement and spontaneous arterio-venous circuits may lose more thermal energy, thus contributing to comparably greater cooling than post-dilutional fluid replacement or pump-driven veno-venous extracorporeal circuits, respectively.

Krouzecky and colleagues only applied regional blood cooling of extracorporeal circuit in their experimental model for a short duration. Additional investigations using a longer duration of CRRT with regional blood cooling in their model are needed to evaluate both efficacy and effectiveness, but also importantly safety, specifically in terms of temperature-induced hemolysis with repeated blood cooling and re-warming. Further, the practical use of TEG for evaluating extracorporeal circuit patency and risk for clotting should be prospectively examined [22]. In addition, the authors aggressively cooled the blood in their experimental porcine model extracorporeal circuit down to $20^{\circ} \mathrm{C}$. Accordingly, there was need to ensure that the blood was fully re-warmed prior to return in the venous access port. Further studies are therefore required to determine whether a similar reduction in extracorporeal clotting and delay in clot fibrin formation also occur at more modest hypothermic temperatures, which may not require the added complexity of careful re-warming of blood prior to return to the patient.

Conflict of interest statement The authors have no conflicts of interest to declare.

\section{References}

1. Davenport A (2004) Anticoagulation for continuous renal replacement therapy. Contrib Nephrol 144:228-238

2. Joannidis M, Oudemans-van Straaten HM (2007) Clinical review: patency of the circuit in continuous renal replacement therapy. Crit Care 11:218

3. Davenport A (1997) The coagulation system in the critically ill patient with acute renal failure and the effect of an extracorporeal circuit. Am J Kidney Dis 30:S20-S27
4. Bouman CS, de Pont AC, Meijers JC, Bakhtiari K, Roem D, Zeerleder S, Wolbink G, Korevaar JC, Levi M, de Jonge E (2006) The effects of continuous venovenous hemofiltration on coagulation activation. Crit Care 10:R150
5. Salmon J, Cardigan R, Mackie I, Cohen SL, Machin S, Singer M (1997) Continuous venovenous haemofiltration using polyacrylonitrile filters does not activate contact system and intrinsic coagulation pathways. Intensive Care Med 23:38-43

6. Cardigan RA, McGloin H, Mackie IJ, Machin SJ, Singer M (1999) Activation of the tissue factor pathway occurs during continuous venovenous hemofiltration. Kidney Int 55:15681574 
7. Holt AW, Bierer P, Bersten AD, Bury LK, Vedig AE (1996) Continuous renal replacement therapy in critically ill patients: monitoring circuit function. Anaesth Intensive Care 24:423-429

8. Cutts MW, Thomas AN, Kishen R (2000) Transfusion requirements during continuous veno-venous haemofiltration: the importance of filter life. Intensive Care Med 26:1694-1697

9. Baldwin I, Bellomo R, Koch B (2004) Blood flow reductions during continuous renal replacement therapy and circuit life. Intensive Care Med 30:2074-2079

10. Aslam AF, Aslam AK, Vasavada BC, Khan IA (2006) Hypothermia: evaluation, electrocardiographic manifestations, and management. Am J Med 119:297-301

11. Schreiber MA (2005) Coagulopathy in the trauma patient. Curr Opin Crit Care 11:590-597

12. Urrea C, Danton GH, Bramlett HM, Dietrich WD (2004) The beneficial effect of mild hypothermia in a rat model of repeated thromboembolic insults. Acta Neuropathol 107:413-420

13. Kheirabadi BS, Delgado AV, Dubick MA, Scherer MR, Fedyk CG, Holcomb JB, Pusateri AE (2007) In vitro effect of activated recombinant factor VII (rFVIIa) on coagulation properties of human blood at hypothermic temperatures. J Trauma 63:1079-1086
14. Viuff D, Lauritzen B, Pusateri AE, Andersen S, Rojkjaer R, Johansson PI (2008) Effect of haemodilution, acidosis, and hypothermia on the activity of recombinant factor VIIa (NovoSeven). Br J Anaesth 101:324331

15. Dunkley S, Phillips L, McCall P, Brereton J, Lindeman R, Jankelowitz G, Cameron P (2008) Recombinant activated factor VII in cardiac surgery: experience from the Australian and New Zealand Haemostasis Registry. Ann Thorac Surg 85:836-844

16. Krouzecky A, Chvojka J, Sykora R, Jaroslav Radej J, Karvunidis T, Novak I, Ruzick J, Petrankova Z, Benes J, Bolek L, Matejovic M (2008) Regional cooling of the extracorporeal blood circuit: a novel anticoagulation approach for renal replacement therapy? Intensive Care Med. doi: 10.1007/s00134-008-1271-9

17. Frank RD, Weber J, Dresbach H, Thelen H, Weiss C, Floege J (2001) Role of contact system activation in hemodialyzer-induced thrombogenicity. Kidney Int 60:1972-1981
18. Wolberg AS, Meng ZH, Monroe DM 3rd, Hoffman M (2004) A systematic evaluation of the effect of temperature on coagulation enzyme activity and platelet function. J Trauma 56:12211228

19. Davenport A (2003) Pre-dilution or post-dilution fluid replacement for continuous veno-venous hemofiltration: that is the question. Nephron Clin Pract 94:c83-c84

20. Bellomo R, Teede H, Boyce N (1993) Anticoagulant regimens in acute continuous hemodiafiltration: a comparative study. Intensive Care Med 19:329-332

21. Davenport A, Will EJ, Davison AM (1994) Comparison of the use of standard heparin and prostacyclin anticoagulation in spontaneous and pump-driven extracorporeal circuits in patients with combined acute renal and hepatic failure. Nephron 66:431-437

22. Holt AW, Bierer P, Glover P, Plummer JL, Bersten AD (2002) Conventional coagulation and thromboelastograph parameters and longevity of continuous renal replacement circuits. Intensive Care Med 28:1649-1655 